\title{
Osteonal Structure in the Equine Third Metacarpus
}

\author{
R. B. MARTIN, ${ }^{1}$ V. A. GIBSON, ${ }^{1}$ S. M. STOVER,${ }^{2}$ J. C. GIBELING,${ }^{3}$ and L. V. GRIFFIN ${ }^{3}$ \\ 1 Orthopedic Research Laboratory, School of Medicine, ${ }^{2}$ Veterinary Orthopedic Research Laboratory, School of Veterinary Medicine, \\ and ${ }^{3}$ Department of Chemical Engineering and Materials Science, College of Engineering, University of California at Davis, Davis, CA, USA
}

\begin{abstract}
In studying the flexural fatigue properties of the equine third metacarpal (cannon) bone, we previously found that the dorsal region was weaker monotonically, but more fatigue resistant, than the lateral region. Fatigue resistance was associated with fracture surfaces which demonstrated that secondary osteons had "pulled out" of the surrounding matrix; this never happened in lateral specimens. We therefore became interested in the osteonal structure of this bone, and began to study its birefringence patterns in circularly polarized light. We found that the predominant type of secondary osteon was one in which only the outermost few lamellae were circumferential, with the inner lamellae being longitudinally oriented. This "hoop" pattern had not been described in Ascenzi's classic papers. Using basic fuchsin-stained, undecalcified cross-sections from the dorsal, medial, and lateral midshaft regions of 12 pairs of cannon bones, we classified 360 secondary osteons according to their birefringence patterns, and measured their inner and outer diameters. We found that variants of the hoop category comprised $60 \%$ of all osteons, but were significantly less common in the dorsal region, where the predominant types were Ascenzi's "Iongitudinal" or "alternating" patterns. The dorsal region also had smaller osteons $(O D=156 \pm 19 \mu \mathrm{m})$ than the medial $(179 \pm 13 \mu \mathrm{m}, p$ $=0.0004)$ and lateral $(182 \pm 13 \mu \mathrm{m}, p=0.0001)$ regions. We postulate that these regional variations in osteonal size and structure, which are obviously produced by regional variations in remodeling, have important mechanical implications. (Bone 19:165-171; 1996)
\end{abstract}

\section{Introduction}

The lamellar structure of bone in general, and of secondary osteons in particular, is of great interest because of its bearing on mechanical properties in relation to aging. There has been considerable debate regarding the nature of the ultrastructural features which produce the lamellar appearance of bone when a cross section is viewed in the light microscope. The oldest school of thought has held that collagen fibers are parallel to one another in each lamella, and there are distinct changes in collagen fiber orientation from one lamella to the next. ${ }^{9,10} \mathrm{Smith}^{30}$ defined three osteonal classifications based on relative amounts of longitudinal and circumferential collagen and the amount of lamellation which was apparent in decalcified sections (without the use of polarized light). Another interpretation is that bone's collagen fibers are laid down in a helicoidal arrangement, so that their orientation rotates very slightly from one fiber layer to the next, and always in the same direction. ${ }^{12}$ This would produce lamellae whose width depends on the thickness of the layer of fibers in which the orientation angle rotates $180^{\circ}$. Still others have argued that the lamellar appearance is caused by variations in the density of the calcified matrix rather than collagen orientation. ${ }^{15,26,28,29,33}$ Each of these three basic views is supported by experimental observations, and they may not be mutually exclusive.

A common way to visualize collagen fiber orientation in bone and other tissues under the light microscope is to use polarized light. When plane polarizing filters are crossed to produce a "dark field," regions in which the fibers are perpendicular to the path of the light (i.e., lie in the plane of the section) appear bright, or birefringent. Conversely, regions in which the fibers are parallel to the light path (i.e., are perpendicular to the plane of the section) appear dark. Applying this technique to cross sections of cortical bone, Ascenzi and Bonucci ${ }^{1,2}$ described three general categories of secondary osteons. Bright osteons are those in which in which the fibers are predominantly transversely or circumferentially oriented, and dark osteons comprise another group in which the fibers are mostly longitudinally oriented. The third category is one in which the fiber orientation changes from lamella to lamella, producing a pattern of alternating light and dark layers within the osteon.

This division of osteons into three types has been criticized as ignoring the fact that osteons are somewhat crooked, and the alignment of osteons relative to the bone axis changes along their length. ${ }^{3,4}$ Thus, birefringence of the fibers is a function of the local orientation of the osteonal axis, as well as any intrinsic organization of its fibers relative to the axis. In plane polarized light, birefringence is also a function of the orientation of the section with respect to the polarization plane. This effect superimposes artifactual "iron cross" extinction patterns on the osteons, but this artifact can be eliminated by using circularly polarized light. ${ }^{6}$ When this is done, the birefringence variations due to lamellar structure remain.

We have examined sections of osteonal bone from humans, dogs, rhesus monkeys, cows, and horses in plane and circularly polarized light. It seems to us that the birefringence patterns of osteons are usually much more varied than the three types described by Ascenzi and Bonucci. While it is possible to find examples of bright, dark, and alternating osteons, it is also clear 
that most osteons do not fit neatly into this scheme (Figure 1). For example, inner lamellae may be bright and outer lamellae dark, or vice versa. Thus, while the thesis that lamellar organization varies from osteon to osteon, producing variations in birefringence and mechanical properties, remains plausible and important, osteonal structures seem to be more complex than those described by the model of Ascenzi and Bonucci.

A related group of experiments has measured the relative birefringence of osteonal bone over entire regions of a cortical cross section. ${ }^{5,6,22-25,27}$ These studies show that there are regional variations in average collagen fiber orientation, and these variations seem to correlate with the kind of mechanical stress that the region habitually experiences. For example, regions in which the usual direction of bending produces greater compression contain fewer longitudinal fibers than regions where bending produces less compression or net tension. Similar studies in machined mechanical test specimens show that average collagen fiber orientation is as well correlated with continuum level mechanical properties as porosity or mineral content. ${ }^{16.17}$ Recently, we applied similar techniques in our attempts to develop a better understanding of the mechanical properties of the equine third metacarpal bone. ${ }^{18}$ We found that the average collagen fiber orientation is more longitudinal in the dorsal than in the lateral portion of the cortex. This was interesting because we have also found that the dorsal region has better fatigue resistance, but is less stiff and monotonically weaker, than the lateral region.

In the course of making these measurements, we observed that many of the equine secondary osteons display a characteristic birefringence pattern which is different from the three types described by Ascenzi and Bonnuci: for the most part of the osteons' lamellae are dark, but the outermost few lamellae are highly birefringent (Figure 2). We called this pattern "hoop birefringence" because it implies that the osteons are characterized by a "hoop" of circumferentially oriented outer lamellae wrapped about an inner bundle of longitudinally oriented lamellae. This kind of structure would seem to have implications with respect to the mechanical properties of the osteons and their interface with the surrounding tissue. This in turn could have something to do with cement line shear strength and the phenomenon of osteonal pullout, which we have determined to be an important factor in fatigue resistance. ${ }^{31}$ Therefore, we decided to study the extent to which this pattern prevailed in the equine metacarpus, and whether or not it varied regionally. Since the tendency for osteons to pull out is theoretically a function of their diameter, ${ }^{21}$ we also asked whether an osteon's size was related to its location in the cortex or its birefringence pattern.

\section{Methods}

The specimens had originally been prepared for the purpose of studying fatigue damage in bone from the equine third metacarpal or "cannon" bone. The mechanical" and histologic ${ }^{19}$ methods have been previously described. Briefly, 36 beams measuring $100 \times 10 \times 4 \mathrm{~mm}$ were machined from the diaphyses of six cannon bone pairs obtained at necropsy from Thoroughbred and non-Thoroughbred racehorses. Five of these animals were females and one was male; the age range was $2-5$ (mean $=3.7$ )

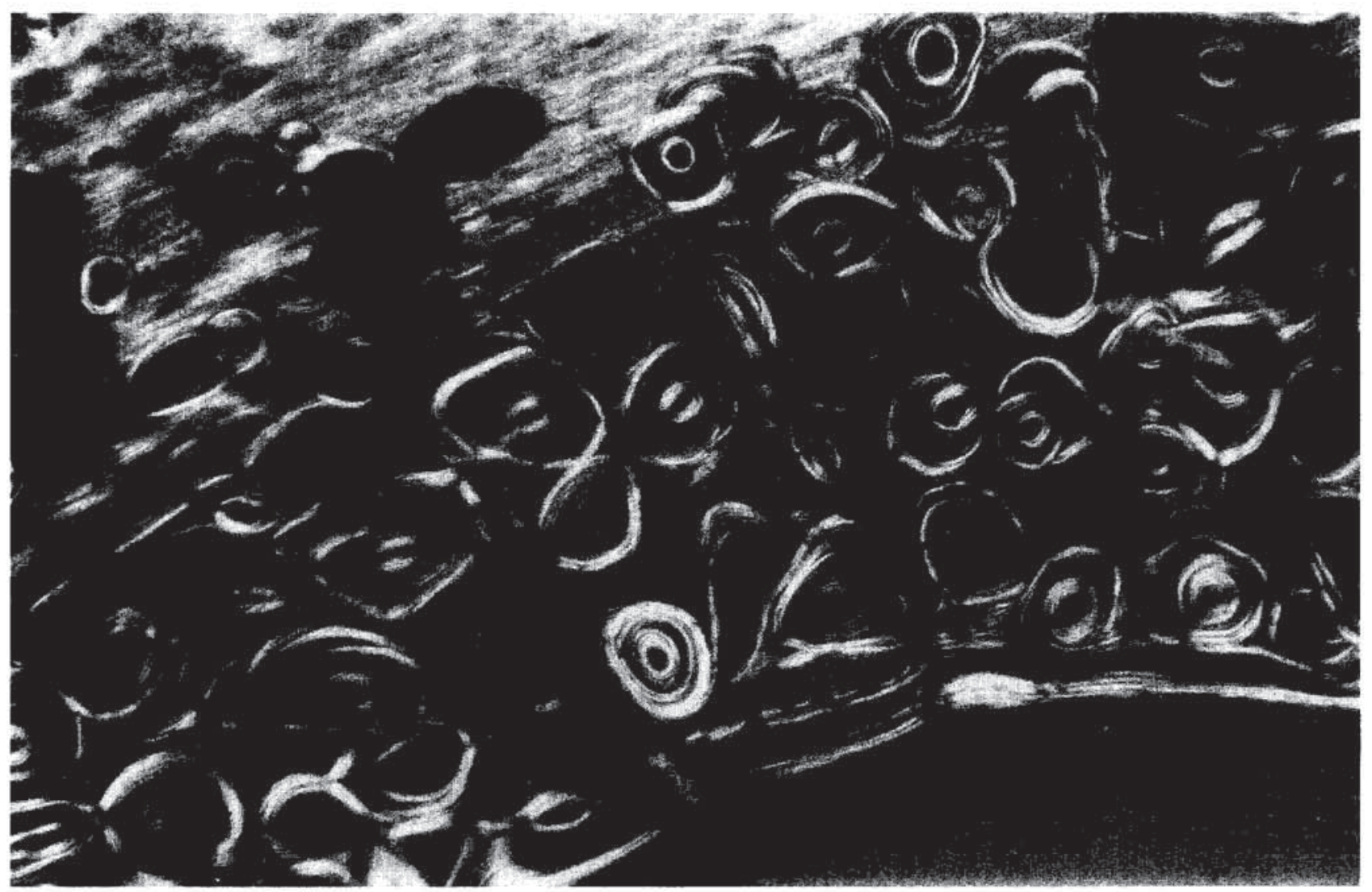

Figure 1. Low power photomicrograph of osteonal bone from the femur midshaft of a rhesus monkey showing normal variability in the birefringence patterns of secondary osteons. The medullary canal is at lower right. Undecalcified section, $100 \mu \mathrm{m}$ thick, stained with tetrachrome, and photographed in circularly polarized light at $10 x$. 


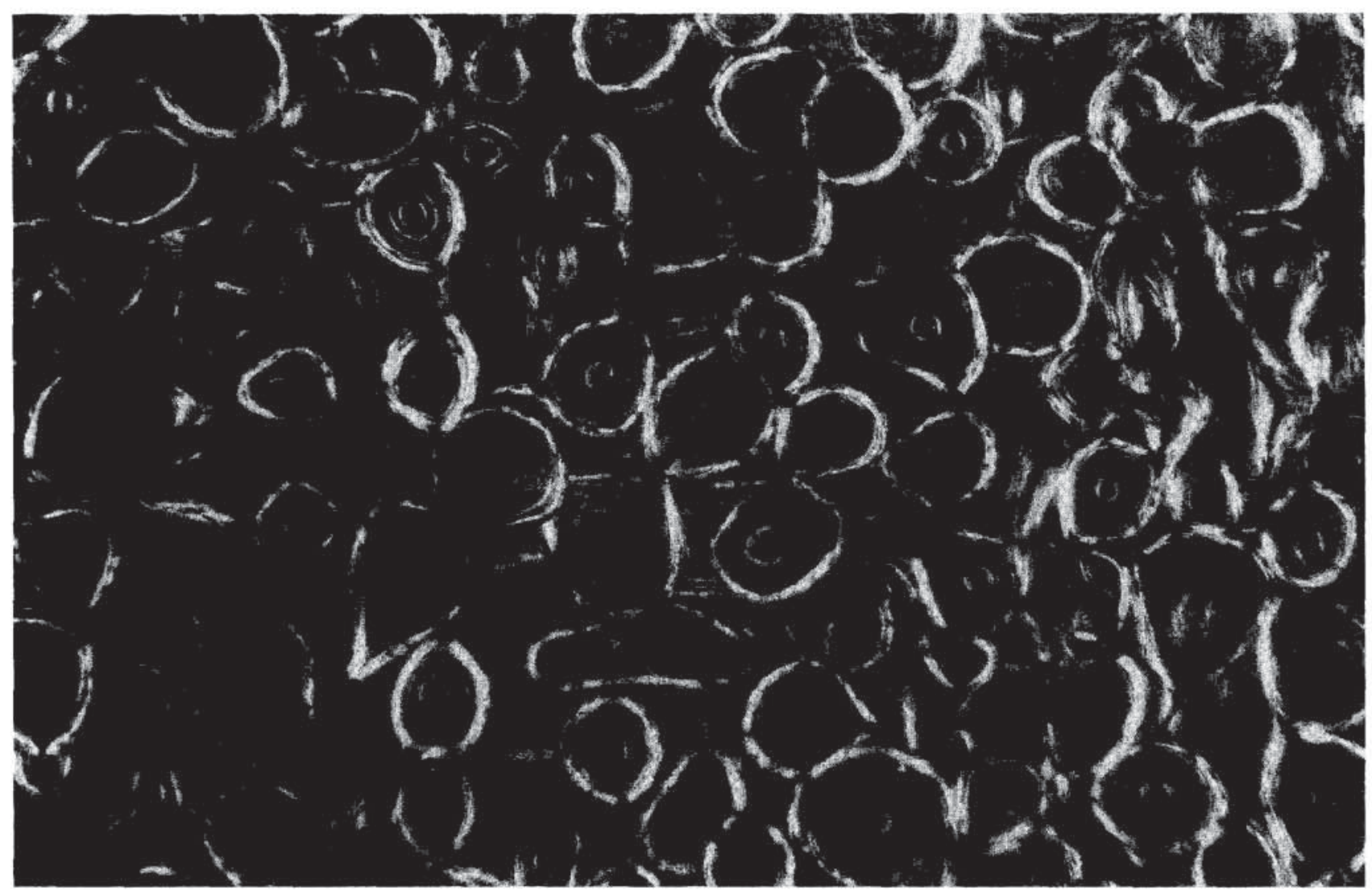

Figure 2. Low power photomicrograph of osteonal bone from the third metacarpal bone of a racehorse showing the characteristic birefringence pattern of its secondary osteons. Usually, most of the lamellae in each osteon are dark, while the outer few lamellae are highly birefringent. Undecalcified section, $100 \mu \mathrm{m}$ thick, stained en bloc with basic fuchsin and photographed in circularly polarized light at 10x.

years. One beam was machined from each of the three thickest cortices of each bone: lateral (L), medial (M), and dorsal (D). The 36 beams were uniformly distributed among three experimental groups. All beams were loaded to fracture in four point bending while immersed in a saline bath at $37^{\circ} \mathrm{C}$, some monotonically and others in fatigue.

Following failure, both fragments of the beam were bulk stained in $1 \%$ basic fuchsin using a modification of the method of Burr and Stafford. ${ }^{3}$ Using a Gillings-Hamco diamond saw, complete cross sections $(10 \times 4 \mathrm{~mm})$ were cut from the center of the beam, ground to a thickness of $100 \pm 5 \mu \mathrm{m}$, and mounted on glass slides with Eukitt (Calibrated Instruments, Inc., Hawthorne, NY).

One section from each beam was examined at $400 \mathrm{X}$ using an Olympus Vanox microscope equipped with circularly polarizing filters below and above the stage. These filters were made by combining a plane polarizing filter with a quarter-wave plate. ${ }^{6}$ With the substage circular polarizer absent so as to render birefringence impossible, and starting at one end of the $10 \times 4 \mathrm{~mm}$ cross section, the specimen was scanned in the $4 \mathrm{~mm}$ direction until a secondary osteon suitable for measurement was found. The selection criteria were (1) presence of a scalloped cement line, (2) absence of a Volkmann's canal connection, (3) reasonably circular or ellipsoidal shape, and (4) refilling complete or nearly so. When the first such osteon was found, its outer (cement line) and inner (Haversian canal) diameters were measured using the linear scale of a Merz eyepiece reticule. This measurement was estimated to within $\pm 3 \mu \mathrm{m}$. The reticule was then rotated $90^{\circ}$ and the diameter measurements were repeated. In all cases the diameter measurements were made through the approximate center of the Haversian canal. Next, the illumination was changed to circularly polarized light by inserting the substage polarizer, and the osteon was classified into one of six categories with respect to its birefringence pattern (Figure 3). The illumination was then returned to ordinary light, and the stage was moved $1 \mathrm{~mm}$ in the $10 \mathrm{~mm}$ direction to set up another scan line. This line was then scanned for the first qualifying osteon, and it was measured and categorized. The process was repeated until data had been collected on ten quasirandomly chosen osteons distributed throughout the section. It is important to understand that it was impossible to predict the birefringence pattern of the osteons in ordinary light; therefore, selection of the osteons was not biased with respect to any particular birefringence pattern. Also, by choosing the first eligible osteon encountered in a scan using only the above criteria, without regard to other characteristics, we attempted to avoid bias with respect to size and other osteonal qualities. All measurements were made by one investigator (RBM).

Inner and outer diameters were analyzed using three factor analysis of variance (ANOVA), the factors being cortical region (dorsal, medial, lateral), side (left or right), and horse (six animals). If horse was a significant factor, the analysis was repeated, blocking on horse to control for individual variations. Birefringence pattern data were analyzed in two ways. First, a $3 \times 6$ contingency table was formed between region and birefringence pattern and a chi-squared analysis was used to test for regional differences. Alternatively, each pattern was assigned a numerical score between 5 and 0 according to the degree to which it con- 

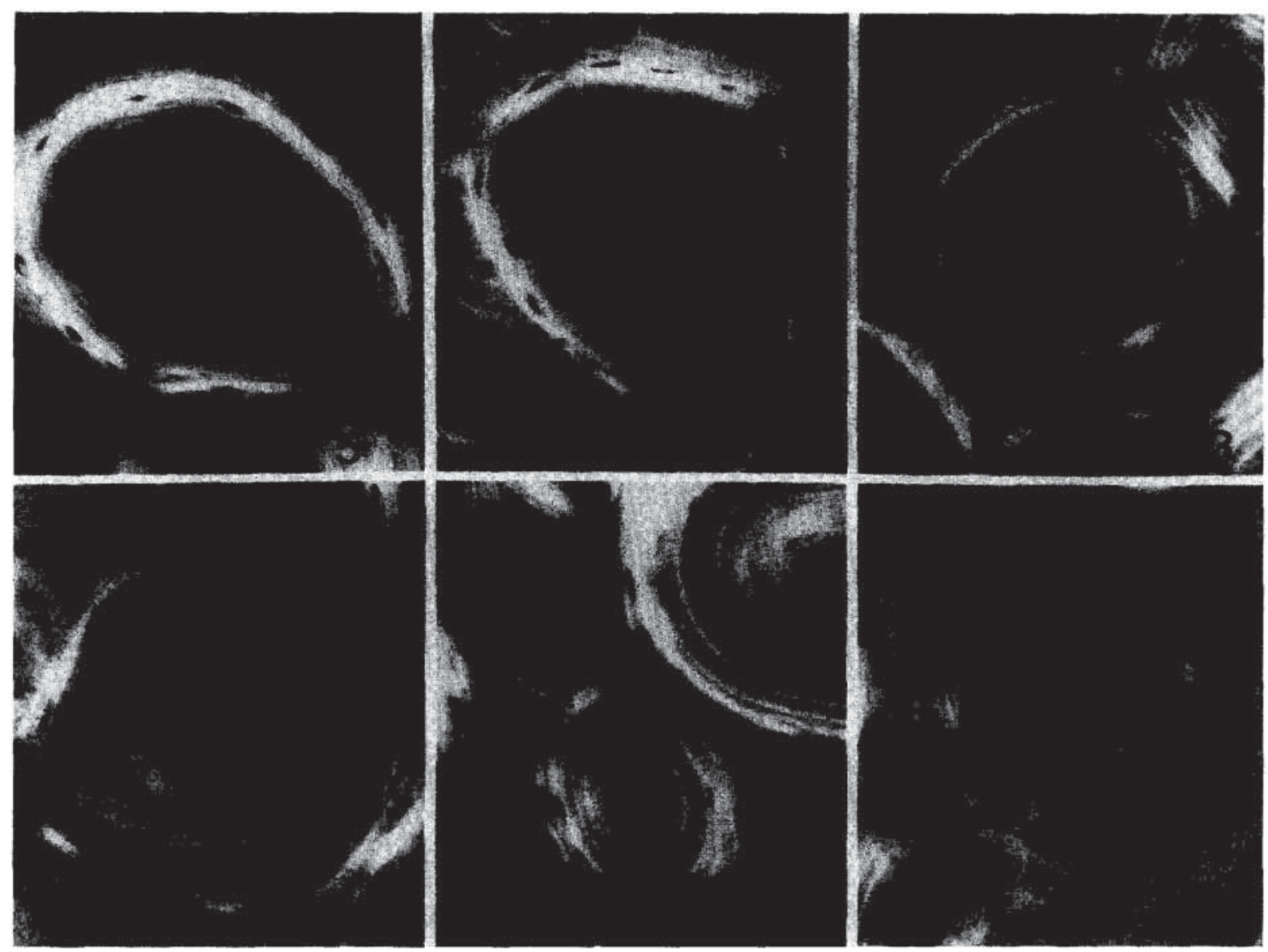

Figure 3. Examples of birefringence patterns in secondary osteons of the equine third metacarpal bone (100 $\mu \mathrm{m}$ thick, undecalcified section, en bloc stained with basic fuchsin). Each photomicrograph in this montage is to the same scale (field width $200 \mu \mathrm{m}$ ) and illustrates one of the six birefringence categories used in the analysis. The numbers labeling the individual images also represent the "hoop score" assigned to that category: $5=$ category $\mathrm{O}$ osteon with dark interior and strongly birefringent peripheral lamellae; $4=$ category OI, similar to $\mathrm{O}$ but the birefringent ring is incomplete; $3=$ category $\mathrm{OW}$, similar to $\mathrm{O}$ but the birefringent ring is weak; 2 = category $\mathrm{OWI}$, a combination of $\mathrm{OI}$ and $\mathrm{OW} ; 1=$ category $\mathrm{D}$, birefringent lamellae are distributed throughout the wall of the osteon; $0=$ category $\mathrm{N}$, a dark osteon with no birefringent lamellae.

formed to the $\mathrm{O}$ category (Figure 3 ). The mean score of the ten osteons studied in each section was calculated to two decimal places and called a "hoop score." The hoop score was treated as a parametric variable, and three way ANOVA was used to study its behavior with respect to cortical region, side, and horse, in the same way as was done for osteon diameters. The relationship between osteon size and birefringence pattern was tested by ANOVA on inner or outer diameter using osteon type and region as factors. The criterion for statistical significance was $p<0.05$, variances are reported as standard deviations, and unreported ANOVA interactions may be assumed not significant.

\section{Results}

Three factor ANOVA showed that osteonal inner (Haversian canal) diameters were independent of region (Table 1), side, and horse. Outer (cement line) diameters were the same in left and right bones, but were significantly affected by region $(p=$ $0.0007)$ and horse $(p=0.0467)$. When the effects of horse were blocked, the analysis showed that the dorsal region had smaller osteons $(156 \pm 19 \mu \mathrm{m})$ than the medial $(179 \pm 13 \mu \mathrm{m}, p=$ $0.0004)$ and lateral $(182 \pm 13 \mu \mathrm{m}, p=0.0001)$ regions, which were not different from one another.
When a chi-squared analysis was done for the regional distribution of the six osteonal categories (Table 2), it was found that the distribution of osteonal types was highly dependent on cortical region $\left(\chi^{2}=103, p<0.001\right)$. The medial region had the most $\mathrm{O}$ osteons, the medial and lateral regions the most $\mathrm{OI}$ and OW osteons, the lateral region the most OWI osteons, and the dorsal region the most $\mathrm{D}$ and $\mathrm{N}$ osteons. The three categories which most strongly manifested the circumferentially wrapped outer lamellae pattern $(\mathrm{O}, \mathrm{OI}$, and $\mathrm{OW})$ comprised $60 \%$ of all the osteons. This percentage was $72 \%$ in the medial and lateral regions combined but only $38 \%$ in the dorsal region, where the most commonly seen osteons were those with birefringent layers distributed throughout their wall.

Table 1. Osteonal diameters and hoop scores by region

\begin{tabular}{lccc}
\hline Region & $\begin{array}{c}\text { Inner diameter } \\
(\mu \mathrm{m})\end{array}$ & $\begin{array}{c}\text { Outer diameter } \\
(\mu \mathrm{m})\end{array}$ & Hoop score \\
\hline Dorsal & $30.9 \pm 6.1$ & $156 \pm 19$ & $2.10 \pm 1.08$ \\
Medial & $33.2 \pm 6.2$ & $179 \pm 13$ & $3.48 \pm 0.41$ \\
Lateral & $31.3 \pm 5.0$ & $182 \pm 13$ & $3.10 \pm 0.59$ \\
Total & $31.3 \pm 4.0$ & $172 \pm 19$ & $2.89 \pm 0.93$ \\
\hline
\end{tabular}


Table 2. Contingency table for regional distribution of osteonal categories

\begin{tabular}{lrrrrrr}
\hline & \multicolumn{5}{c}{ Osteonal categories } \\
\cline { 2 - 7 } Region & O & OI & OW & OWI & D & N \\
\hline Dorsal & 23 & 6 & 16 & 15 & 48 & 12 \\
Medial & 44 & 18 & 32 & 11 & 14 & 1 \\
Lateral & 26 & 14 & 39 & 34 & 2 & 5 \\
Total & 93 & 38 & 87 & 60 & 64 & 18 \\
\hline
\end{tabular}

These results were supported by the "hoop score" method of analyzing the data. Three factor ANOVA showed that hoop scores were the same in left and right bones but were significantly affected by region $(p=0.0002)$ and horse $(p=0.0441)$. There was also a significant side-region interaction $(p=$ 0.0205 ). When the effects of horse were blocked, the analysis showed that the dorsal region had smaller hoop scores $(2.10 \pm$ $1.08)$ than the lateral $(3.10 \pm 0.59, p=0.0009)$ and medial $(3.48$ $\pm 0.41, p<0.0001)$ regions, which were not different from one another. The side-region interaction persisted with horse blocked ( $p=0.0416$, Figure 4). Generally speaking, however, the dorsal hoop scores were less than the lateral and medial scores (Table 3).

The relationship between osteon size and birefringence pattern was first examined by tabulating the outer and inner diameter data for all 360 measured osteons by osteon type and cortical region. The diameters of those osteons in each cell which came from the same horse were averaged together to obtain independent samples. Considering first the outer diameters (Figure 5), ANOVA showed that osteon type was significant $(p<0.0001)$, but region was not ( $p=0.142)$; however, there was a significant interaction between these two factors $(p=0.0159)$. The various differences between subgroups are too numerous to describe, but basically osteons without birefringence (type $\mathrm{N}$ ) were smaller than the other categories (Figure 5). Averaging over region, the outer diameter of the OI osteons was greatest at $199.4 \pm 29.1 \mu \mathrm{m}$, while that of the $\mathrm{N}$ osteons was least at $107.4 \pm 30.0 \mu \mathrm{m}$. Similar ANOVA on the osteons' inner diameters showed again that the birefringence pattern was significant $(p=0.0242)$, but region was not $(p=0.683)$; however, this time there was no interaction $(p=0.898)$. The Haversian canals of $\mathrm{N}$ osteons were smallest $(26.9 \pm 4.7 \mu \mathrm{m})$ and were significantly smaller than those of $\mathrm{O}$ and OI osteons ( $p<0.0275$ and 0.0008 , respectively). Haversian

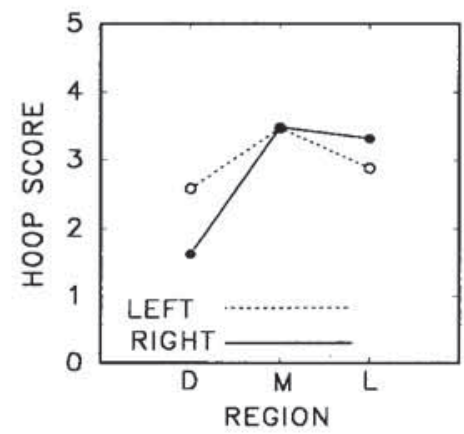

Figure 4. Hoop scores by region and side. Error bars omitted for clarity; see Table 3 for statistically significant differences. It is conceivable that the side-region interaction arises from the fact that the specimens came from racehorses which habitually race and do their hard workouts in a counterclockwise direction.
Table 3. Side-region interactions for hoop score effects in Figure 4

\begin{tabular}{llr}
\hline Subgroup & Different from ... & $p$-value \\
\hline Left dorsal & Left medial & 0.0004 \\
& Right lateral & 0.0188 \\
Right dorsal & Left medial & 0.0107 \\
& Left lateral & 0.0003 \\
Left lateral & Right lateral & $<0.0001$ \\
& Right medial & 0.0001 \\
\hline
\end{tabular}

canals of OI osteons were largest $(36.6 \pm 7.2 \mu \mathrm{m})$ and were larger than those of all other categories except $O(p<0.05$ in all cases).

\section{Discussion}

In these experiments, we studied the extent to which secondary osteons in the equine third metacarpal bone were characterized by hoop birefringence: a few peripheral lamellae which were birefringent, and a nonbirefringent interior. We also asked whether the numbers of osteons with this feature varied by cortical region. We found that hoop birefringence was the dominant osteonal type in the medial and lateral regions of the cortex, but not in the dorsal region. There, a plurality of the sampled osteons had birefringent lamellae distributed throughout their wall. We also asked whether the inner and outer diameters of secondary osteons varied regionally or were related to the birefringence patterns. We found that there were interactions between these two factors, but in general osteons with no birefringence at all had smaller inner and outer diameters than those with hoop birefringence.

There are several limitations to this study. First, it has been assumed that mechanical loading did not alter the collagen fiber orientations or birefringence patterns in the specimens, which had been fatigued to failure. This assumption is supported by the fact that bone is a brittle, calcified tissue which fails by crack propagation rather than macroscopic plastic flow. There is no evidence that, and it would be difficult to imagine how, the lamellar structure of individual osteons throughout the specimen could be distorted by mechanical loading so as to convert, say, type $\mathrm{O}$ osteons to type $\mathrm{D}$ or $\mathrm{N}$ osteons.

Another limitation is the subjective judgment involved in

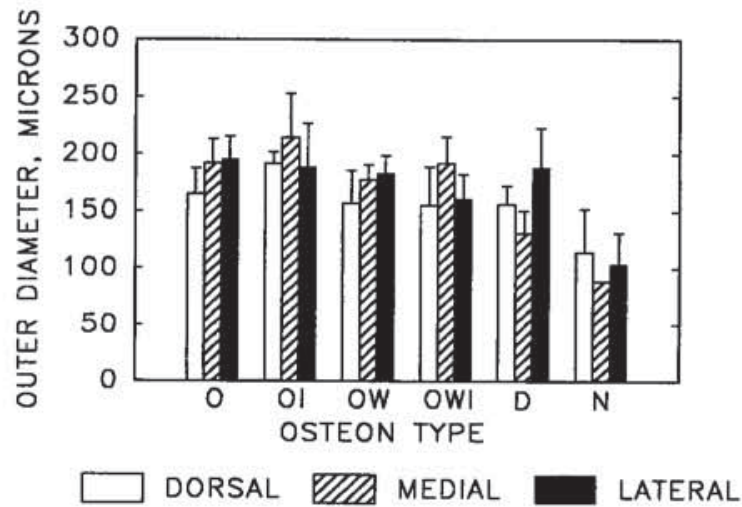

Figure 5. Cement line (outer) diameters of osteons by region and type. Error bars show standard deviations. Only one type $\mathrm{N}$ osteon was encountered in the medial portion of the sample. Hence, this datum has no error bar. 
classifying the osteons. However, the work was all done by one investigator who was blinded to the identity of the specimens. There was also a "practice period" in which ten specimens (100 osteons) were measured before undertaking the sample reported here. Most of the osteons were clearly members of a particular category, but a few were mixtures. For example, sometimes a type $\mathrm{O}$ osteon, with a strong peripheral "hoop," would also have a substantial number of birefringent lamellae in its interior as well. If so, it was given the D classification. In other cases, type $\mathrm{O}$ osteons had a single additional birefringence hoop at the margin of the Haversian canal. These were placed in the $\mathrm{O}$ category because most of the lamellae were non-birefringent.

A more important limitation derives from the fact that the literature contains several different interpretations of bone's birefringence behavior in relationship to its lamellar structure, and there is serious debate about the nature of the lamellar structure itself. While a number of laboratories have published studies based on the principle that birefringence arises from collagen fiber orientation in bone $e^{1.2 .5}, 12,16,17.22-25.27$ and other tissues, ${ }^{14,20}$ alternative views are supported by other means of investigation. Particularly noteworthy is the recent assertion by Marotti ${ }^{15}$ that bone lamellae are not defined by changes in collagen fiber orientation, but rather by regular variations in the density of the matrix. Marotti postulates that all lamellae, both "dense" and "loose," contain collagen fibers which weave about in various directions. He further asserts that the dense lamellae are birefringent not because of the orientation of their fibers, but simply because they contain more fibers, including those which happen to be parallel to the plane of the section and produce birefringence. Examination of his photomicrographs and those of other investigators who interpret lamellar structure differently (e.g., Refs. 6, 12, and 30) indicates that they are seeing the same structural configurations but interpreting them differently. We would agree with Marotti's view that it is incorrect to think of bony lamellae as precisely formed layers of perfectly parallel collagen fibers, but we also believe that he has overlooked significant variations in average fiber orientation between what he calls loose and dense lamellae.

Regardless of which of these schools of thought is more correct, we believe that the results reported here are important. Marotti ${ }^{15}$ himself agrees that the use of circularly polarized light to elicit information about variations in lamellar structure is "on the whole acceptable" (pp. S51-S52). What needs to be kept in mind at this point is that both the structural and mechanical implications of the birefringence observations remain to be determined. When we embarked on this study, we thought perhaps the peripheral hoop of circumferential collagen fibers in the $\mathrm{O}$ category osteons would allow them to debond bore easily from the surrounding matrix, enhancing osteon "pullout" and fatigue life. Thus, we expected to see more O,OI, OW, and OWI osteons in the dorsal region, where the most osteon pullout and longest fatigue lives were observed. ${ }^{31}$ In fact, we have seen the opposite distribution: The dorsal region contains the most osteons with birefringent lamellae distributed through the wall, or no birefringence at all. This might imply that "hoop" osteons are more tightly bound to the surrounding bone, and less likely to pull out, but increase the elastic modulus and confer greater monotonic strength. Alternatively, the circumferential fibers in the outer lamellae may enhance the behavior of the cement line as a barrier to microcracks. Carter and Hayes ${ }^{8}$ observed that microcracks cross cement lines more often under compressive loading than under tension. In vivo strain gauge data indicate that the medial and lateral cortices lie on the compressive side of the neutral axis while dorsal cortex alone is in tension during the most stressful part of the racehorse's gait cycle. ${ }^{13}$ Thus, it would make sense for osteons in the habitually compressed medial and lateral regions to have "hoops" if this provides greater protection against microcrack invasion. We are pursuing these microstructural and mechanical issues analytically and experimentally.

Finally, we would like to briefly comment on our osteonal classification scheme in relation to the classic works of Ascenzi and Bonucci, ${ }^{1.2}$ which described three types of osteons. Clearly, our type $\mathrm{N}$ and $\mathrm{D}$ osteons are synonymous with their dark and alternating osteons, respectively. We did not observe Ascenzi and Bonucci's bright type (having a completely birefringent wall) in our equine specimens, but saw many peripherally birefringent osteons instead (Figure 2). Reexamination of sections of bovine, canine, monkey, and human bone available in our laboratories indicated that type $\mathrm{O}$ osteons and their variants are not infrequent in these species as well. For example, Figure 1 shows several examples of type $\mathrm{O}$ osteons in the femur of a rhesus monkey, including the variant having a hoop abutting the Haversian canal as well as the cement line. We would like to suggest that this kind of osteonal structure, in combination with Ascenzi's alternating and dark osteons, completes a set of three commonly seen osteonal structures. These types of osteons comprise a set of microstructural building blocks conferring diverse mechanical properties on moieties of cortical bone, appropriate to the stresses which they experience. It is essential that we learn the microstructures associated with these osteonal types so that we gain a better understanding of their function in the mechanical adaptation of cortical bone.

\section{References}

1. Ascenzi, A. and Bonucci, E. The compressive properties of single osteons. Anat Rec 161:377-391; 1968

2. Ascenzi, A. and Bonucci, E. The tensile properties of single osteons. Anat Rec 158:375-386; 1967

3. Black, J., Richardson, S. P., and Mattson, R. U. Haversion osteons: Longitudinal variation of internal structure. J Biomed Mater Res 14:41-53; 1980.

4. Black, J., Mattson, R., and Korostoff, E. Haversian osteons: Size, distribution, internal structure. J Biomed Mater Res 8:299-318; 1974.

5. Boyde, A. and Riggs, C. M. The quantitative study of the orientation of collagen in compact bone slices. Bone 11:35-39; 1990.

6. Boyde, A., Bianco, P., Portigliatti-Barbos, M., and Ascenzi, A. Collagen orientation in compact bone: I. A new method for the determination of the proportion of collagen parallel to the plane of compact bone sections. Metab Bone Dis Relat Res 5:299-307; 1984.

7. Burr, D. B. and Stafford, T. Validity of the bulk-staining technique to separate artifactual from in vivo bone microdamage. Clin Orthop Relat Res 260:305308; 1990.

8. Carter, D. R, and Hayes, W. C. Compact bone fatigue damage: a microscopic examination. Clin Orthop Relat Res 127:265-274; 1977.

9. Ebner, V. V. Uber den feineren bau der skeletteile der kalkschwamme usw. Wiener Sitzber 95:213-236; 1887

10. Gebhardt, W. Uber funktionell wichtige anordnungsweisen der feineren und groberen bauelemente des wirbeltierknochens. II. Spezieller teil. Der bau der Haversschen lamellensyteme und seine funktionelle bedeutung. Arch Entwickl Mech Org 20:187-322; 1906.

11. Gibson, V. A., Stover, S. M., Martin, R. B., Gibeling, J. C., Gustafson, M. B., and Griffin, L. V. Fatigue behavior of the equine third metacarpus: mechanical property analysis. J Orthop Res 13:861-868; 1995. 
12. Giraud-Guille, M. M. Twisted plywood architecture of collagen fibrils on human compact bone osteons. Calcif Tiss Int 42:167-180; 1988.

13. Gross, T. S., McLeod, K. J., and Rubin, C. T. Characterizing bone strain distributions in vivo using three triple rosette strain gages. J Biomechan 25:10811087; 1992.

14. Junqueira, L. C. U., Bignolas, G., and Brentani, R. R. Picrosirius staining plus polarization microscopy, a specific method for collagen detection in tissue sections. Histochem J 11:447-455; 1979.

15. Marotti, G. A new theory of bone lamellation. Calcif Tiss Res 53:S47-S56; 1993.

16. Martin, R. B. and Boardman, D. L. The effects of collagen fiber orientation. porosity, density, and mineralization on bovine cortical bone bending properties. J Biomechan 26:1047-1054; 1993.

17. Martin, R. B. and Ishida, J. The relative effects of collagen fiber orientation. porosity, density, and mineralization on bone strength. J Biomechan 22:419426; 1989.

18. Martin, R. B., Lau, S. T., Mathews, P. V., Gibson, V. A., and Stover, S. M. Collagen fiber organization is related to mechanical properties and remodeling in equine bone. A comparison of two methods. $\mathbf{J}$ Biomech. In press.

19. Martin, R. B., Stover, S. M., Gibson, V. A., Gibeling, J. C., and Griffin, L. V. Fatigue behavior of the equine third metacarpus: Remodeling and microcrack damage analysis. Unpublished.

20. Neville, A. C. In: Biology of Fibrous Composites. Development beyond the Cell Membrane. Cambridge, England: Cambridge University Press; 1993.

21. Pope, M. H. and Murphy, M. C. Fracture energy of bone in a shear mode. Med Biol Eng 12:763-767; 1974.

22. Portigliatti-Barbos, M., Carando, S., Ascenzi, A., Boyde, A., and Improta, S. A biomechanical analysis at lamellar level of femoral shafts deformed in bending. In: Bergmann, G., Kobel, R., and Rohlmann, A., Eds. Biomechanics: Basic and Applied Research. Dordrecht: Martinus Nijhoff; 1987.

23. Portigliatti-Barbos, M., Bianco, P., Ascenzi, A., and Boyde, A. Collagen orientation in compact bone: II. Distribution of lamellae in the whole of the human femoral shaft with reference to its mechanical properties. Metab Bone Dis Relat Res 5:309-315; 1984.
24. Portigliatti-Barbos, M., Bianco, P., and Ascenzi. A. Distribution of osteonic and interstitial components in the human femoral shaft with reference to struc ture, calcification and mechanical properties. Acta Anat 115:178-186; 1983.

25. Portigliatti-Barbos, M., Bianco, P., and Ascenzi, A. Structural and biomechanical analysis of osteonic compact bone: A new method. In: Huiskes, R., Campen, D. V., and Wijn, J. D., Eds. Biomechanics: Principles and Applications. The Hague: Martinus Nijhoff; 1982; 261-266.

26. Ranvier, J. Traite Technique d'Histologie. Paris: Savy; 1889.

27. Riggs, C. M., Lanyon, L. E., and Boyde, A. Functional associations between collagen fiber orientation and locomotor strain direction in cortical bone of the equine radius. Anat Embryol [Berl] 187:231-238; 1993

28. Rouiller, C. H., Huber, L., Kellenberger, E. D., and Rutishauser, E. La structure lamellaire de l'osteone. Acta Anatomica 14:9-22; 1952.

29. Ruth, E. B. Bone studies. I. Fibrillar structure of adult human bone. Am J Anat 80:35-54; 1947.

30. Smith, J. W. The arrangement of collagen fibres in buman secondary osteones J Bone Jt Surg 42B:588-605; 1960.

31. Stover, S. M., Martin, R. B., Gibson, V. A., Gibeling, J., and Griffin, L. Osteonal pullout increases fatigue life of cortical bone. Trans Orthop Res Soc 20:129; 1995.

32. Stover, S. M., Pool, R. R., Martin, R. B., and Morgan, J. P. Histologic features of the dorsal cortex of the third metacarpal bone mid-diaphysis during postnatal growth in Thoroughbred horses. J Anat 181:455-469; 1992.

33. Ziegler, O. Studien uber die feinere struktur des rohrenknochens und dessen polarization. Deutsche Zeitschrift fur Chirurgie 85:248-262; 1908. 